PROCEEDINGS OF THE

AMERICAN MATHEMATICAL SOCIETY

Volume 129, Number 10, Pages 3069-3077

S 0002-9939(01)05899-3

Article electronically published on February 22, 2001

\title{
NEGATIVELY PINCHED 3-MANIFOLDS ADMIT HYPERBOLIC METRICS
}

\author{
DALE N. SKINNER
}

(Communicated by Christopher Croke)

\begin{abstract}
We show that any compact 3-manifold carrying a metric with sufficiently pinched negative Ricci curvature admits a hyperbolic metric. This proof is a corrected version of the proof first suggested by Maung Min-Oo. The key insight in this new proof is that the error in Min-Oo's paper does not occur if the type $(4,0)$ curvature is considered instead of the type $(3,1)$ curvature.
\end{abstract}

\section{INTRODUCTION}

Richard Hamilton first introduced the Ricci flow in 1982 [Ha] to aid in determining which compact 3-manifolds carry metrics with constant positive sectional curvature. Since then the Ricci flow has proved a most fruitful area of study. The early results depended heavily on the pointwise maximum principle and all required some type of positive curvature hypothesis.

The first attempt at applying the Ricci flow to metrics with negative curvature on arbitrary dimensional manifolds was by Maung Min-Oo in 1990 [MO]. Given a compact Riemannian manifold, we denote its sectional curvature by $K$, its diameter by $d$, and define the average scalar curvature by

$$
r=\frac{\int_{M} s d V_{g}}{\operatorname{Vol}(M)} .
$$

Min-Oo claimed to prove the following:

Claim A. For $n \geq 3$ and $\Lambda>0$, let $\mathcal{M}^{-}(n, \Lambda)$ denote the collection of all compact Riemannian manifolds $(M, g)$ of dimension $n$ with negative average scalar curvature and $d^{2} \max |K| \leq \Lambda^{2}$. Then for every $n \geq 3$ and $\Lambda>0$ there is a constant $\epsilon>0$, depending just on $n$ and $\Lambda$, such that if $(M, g) \in \mathcal{M}^{-}(n, \Lambda)$ and if

$$
\max _{|X|=1}\left|\frac{n \operatorname{Rc}(X, X)}{r}-1\right|<\epsilon,
$$

then $M$ admits an Einstein metric with Einstein constant -1 .

In the case of 3-manifolds, the claim actually implies the existence of a constant curvature metric. This follows from the well-known result that a metric on a 3manifold is Einstein if and only if it has constant sectional curvature.

Received by the editors March 27, 1997 and, in revised form, February 17, 2000.

2000 Mathematics Subject Classification. Primary 53C20; Secondary 53C21, 53C25, 58J60.

Research supported in part by National Science Foundation grant DMS-9404107. 
Min-Oo's technique was to work with the (slightly modified) Ricci flow

$$
g^{\prime}=-2 \mathrm{Rc}+\frac{2 r_{0}}{n} g,
$$

where $r_{0}$ is the average scalar curvature of the initial metric. The need for a positive curvature assumption is avoided by working with $L^{2}$-estimates instead of pointwise estimates. Unfortunately, there is an error at an early stage in Min-Oo's work. This was first pointed out in 1993 by Rugang Ye [Ye].

There is a topological proof of Claim A for three-manifolds due to Mike Anderson based on Gromov's Precompactness Theorem. Although a quite elegant proof, this approach gives no information on the pinching constant in the theorem. Another proof of the result can be given using the main result of $[\mathrm{Ye}]$ and some subtle estimates on the spectrum of the second order differential operator used in Ye's work. This proof, which was indicated to us by Steven Altschuler, also gives an explicit form for the bound on the size of the pinching constant in terms of geometric quantities. (The constant decays exponentially in $\Lambda$.) The proof presented here is a corrected version of the proof proposed by Min-Oo. This proof, which appears to be new, is much more elementary than Ye's work, completely avoiding the need to consider the second variation of the normalized total scalar curvature.

We are indebted to Mike Anderson for indicating the topological proof to us, and to Steven Altschuler for suggesting that Min-Oo's result might follow from Ye's work. We also wish to thank Jack Lee for his helpful comments on the various drafts of this note and for countless hours of conversation about differential geometry in general, and the Ricci flow in specific.

\section{Preliminaries}

In this section we gather some preliminary results that are needed in the sequel. All manifolds are assumed to be smooth. We observe the summation convention and use the metric $g_{i j}$ and its inverse $g^{i j}=\left(g^{-1}\right)^{i j}$ to lower and raise indices. The pointwise inner products on various tensor bundles induced by a metric $g$ will all be denoted by $\langle\cdot, \cdot\rangle$ and the corresponding $L^{2}$ inner products will be denoted $(\cdot, \cdot)$. On any Riemannian manifold $(M, g)$ the Levi-Civita connection will be denoted by $\nabla$. Components of covariant derivatives of tensors will be denoted by indices preceded by a comma, e.g. the first covariant derivative of a 2 -tensor $\tau$ has components $\tau_{i j, k}$. The divergence of a $k$-tensor $\alpha$ is given in coordinates as $\delta \alpha_{i_{1} \cdots i_{k-1}}=\alpha_{i_{1} \ldots i_{k-1} j}{ }^{j}$.

We follow Hamilton's curvature conventions. The curvature operator on $M$ is defined by $R(X, Y) Z=\nabla_{X} \nabla_{Y} Z-\nabla_{Y} \nabla_{X} Z-\nabla_{[X, Y]} Z$ and is written in coordinates as

$$
R\left(\partial_{i}, \partial_{j}\right) \partial_{l}=R_{i j}{ }^{k} \partial_{k} .
$$

The Riemann tensor $\mathrm{Rm}$ is then given by

$$
R_{i j k l}=g_{k p} R_{i j}{ }^{k}{ }_{l} .
$$

With this convention we have $R_{i j i j}>0$ for the standard spheres. This convention has become fairly standard in the Ricci flow literature. However, the reader must be warned that this is the opposite of the convention used by Min-Oo.

We recall the decomposition of the Riemann tensor into irreducible components. (See Section 1.G of [Be] for details.) Define the Kulkarni-Nomizu product of a pair 
of two-tensors $\sigma$ and $\tau$ by

$$
(\sigma \bigotimes \tau)_{i j k l}=\sigma_{i k} \tau_{j l}+\sigma_{j l} \tau_{i k}-\sigma_{i l} \tau_{j k}-\sigma_{j k} \tau_{i l} .
$$

For any tensor $C \in \Sigma^{2} \Lambda^{2} T^{*} M$, we can define the Ricci tensor $\operatorname{Ric}(C)$ and scalar curvature $\operatorname{Scal}(C)$ by the same formulas as in the case of a Riemannian metric, i.e.

$$
\operatorname{Ric}(C)_{i j}=g^{k l} C_{i k j l} \quad \text { and } \quad \operatorname{Scal}(C)=\operatorname{tr}(\operatorname{Ric}(C)) .
$$

(The symmetries of $C$ imply that $\operatorname{Ric}(C)$ is symmetric.) We denote the Ricci tensor and scalar curvature of a Riemannian metric by Rc and $s$, respectively. If $\Sigma_{0}^{2} M$ denotes the bundle of traceless symmetric 2-tensors, then the following orthogonal decomposition holds:

$$
\Sigma^{2} \Lambda^{2} T^{*} M=\operatorname{ker}(\mathrm{Ric}) \oplus \mathbb{R} g \oslash g \oplus \Sigma_{0}^{2} M \bowtie g .
$$

The decomposition of a tensor $C$ in $\Sigma^{2} \Lambda^{2} T^{*} M$ is

$$
C=W+\underbrace{\frac{\operatorname{Scal}(C)}{2 n(n-1)} g \oslash g}_{K}+\underbrace{\frac{1}{n-2} \operatorname{Ric}(C) \oslash g}_{Z}
$$

where $\operatorname{Ric}(C)$ denotes the traceless Ricci tensor of $C$

$$
\operatorname{Ric}(C)=\operatorname{Ric}(C)-\frac{\operatorname{Scal}(C)}{n} g
$$

and $W$ has zero Ricci tensor. The tensors $W, K$, and $Z$ are called the Weyl tensor, the scalar curvature part of $C$, and the traceless Ricci part of $C$, respectively.

\section{THE PROOF}

We refer to the introduction of $\mathrm{Ye}$ for a discussion of the error in Min-Oo's work, but basically the problem was in his working with the type $(3,1)$ curvature tensor. The problem that arose in Min-Oo's paper does not occur if we consider the Riemann tensor instead of the curvature operator. In this case we have that $\mathrm{Rm}^{\prime}$ lies in the fixed bundle $\Sigma^{2} \Lambda^{2} T^{*} M$. If Pr denotes the operator on $\Sigma^{2} \Lambda^{2} T^{*} M$ which takes the traceless Ricci part, then we have $\left\langle\operatorname{Pr}\left(\mathrm{Rm}^{\prime}\right), Z\right\rangle=\left\langle\mathrm{Rm}^{\prime}, Z\right\rangle$. This observation is key in our proof of Claim A in the three-dimensional case.

Let $h=\mathrm{Rc}-\frac{r_{0}}{n} g$ and $\widetilde{R}=\operatorname{tr} h=s-r_{0}$. (Min-Oo calls $\widetilde{R}$ the hyperbolic scalar curvature.) The key ingredient in the proof of Claim A is an estimate on the evolution of the $L^{2}$-norm of $h$. Although we only need the estimate for the case $n=3$, for future reference we derive it for arbitrary $n$.

Lemma 3.1. Let $g_{0}$ be a metric on a compact $n$-manifold with negative average scalar curvature $r_{0}$. Set $h=\mathrm{Rc}-\frac{r_{0}}{n} g$. Under the evolution equation (1.2) with $g_{0}$ as the initial data, the square of the $L^{2}$-norm of the flow vector $h$ satisfies the following estimate:

$$
\frac{1}{2} \frac{d}{d t} \int|h|^{2} \leq\left(\frac{2}{n}-\frac{1}{n-1}\right) r_{0} \int|h|^{2}+c(n) \int|h|^{3}+\int\langle\stackrel{\circ}{\mathrm{Re}} \otimes \stackrel{\circ}{\mathrm{Rc}}, \widehat{W}\rangle,
$$

where $\widehat{W}_{i j k l}=W_{i k j l}$ and $c(n)$ is a constant depending only on the dimension $n$. 
Proof. We follow the same general outline as in Min-Oo's proof of his equation (2.21), replacing all occurrences of tensors of type $(3,1)$ with the corresponding tensors of type $(4,0)$. In particular, from here on $\operatorname{Pr}$ will denote the operator on $\Sigma^{2} \Lambda^{2} T^{*} M$ which is a projection onto $\Sigma_{0}^{2} M \bowtie g$. Also $W, K$, and $Z$ will always denote, respectively, the Weyl tensor, scalar curvature part, and traceless Ricci part of the Riemann tensor. All Laplacians are rough Laplacians, i.e. $\Delta=\nabla^{*} \nabla$.

From $1.174(\mathrm{e})$ of $[\mathrm{Be}$, the first variation of the scalar curvature is given by

$$
D s_{g}(\sigma)=-\langle\mathrm{Rc}, \sigma\rangle+\nabla^{*} \nabla \operatorname{tr} \sigma+\delta^{2} \sigma .
$$

So by the chain rule the evolution equation for the scalar curvature is

$$
s^{\prime}=D s_{g}\left(g^{\prime}\right)=-2 D s_{g}(\mathrm{Rc})+\frac{2 r_{0}}{n} D s_{g}(g) .
$$

The first term in the sum is just the evolution of the scalar curvature under the unnormalized Ricci flow $g^{\prime}=-2 \mathrm{Rc}$, which is known from Corollary 7.5 of [Ha] to be

$$
s^{\prime}=-\Delta s+2|\operatorname{Rc}|^{2} .
$$

The second term is just a multiple of the scalar curvature since the covariant derivative of $g$ is zero. Thus the scalar curvature evolves by

$$
s^{\prime}=-\Delta s+2|\operatorname{Rc}|^{2}-\frac{2 r_{0} s}{n} .
$$

Note that

$$
|h|^{2}=|\mathrm{Rc}|^{2}-2 \frac{r_{0}}{n}\langle\mathrm{Rc}, g\rangle+\frac{r_{0}^{2}}{n^{2}}|g|^{2}=|\mathrm{Rc}|^{2}+\frac{r_{0}^{2}}{n}-\frac{2 r_{0} s}{n} .
$$

Using this and the evolution equation (3.2) for the scalar curvature we find that

$$
\begin{aligned}
\widetilde{R}^{\prime}+\Delta \widetilde{R} & =s^{\prime}+\Delta s \\
& =2|\mathrm{Rc}|^{2}-\frac{2 r_{0} s}{n} \\
& =2\left(|h|^{2}-\frac{r_{0}^{2}}{n}+\frac{2 r_{0} s}{n}\right)-\frac{2 r_{0} s}{n} \\
& =2|h|^{2}+\frac{2 r_{0}}{n} \widetilde{R} .
\end{aligned}
$$

A simple coordinate computation shows that for any symmetric 2-tensor $\sigma$ we have

$$
|\sigma \bowtie g|^{2}=4(n-2)|\sigma|^{2}+4(\operatorname{tr} \sigma)^{2} .
$$

Substituting the traceless Ricci tensor in for $\sigma$ yields

$$
|Z|^{2}=\frac{4}{n-2}|\stackrel{\circ}{\mathrm{Rc}}|^{2} \text {. }
$$

Using this we compute that

$$
\begin{aligned}
|h|^{2} & =\left|\stackrel{\circ}{\mathrm{R} c}+\frac{s-r_{0}}{n} g\right|^{2} \\
& =|\stackrel{\circ}{\mathrm{R}}|^{2}+\frac{\widetilde{R}^{2}}{n} \\
& =\frac{n-2}{4}|Z|^{2}+\frac{\widetilde{R}^{2}}{n} .
\end{aligned}
$$


So in order to estimate $\int|h|^{2}$, it suffices to estimate $\int \widetilde{R}^{2}$ and $\int|Z|^{2}$.

Using (3.3), (3.5), and the product rule we compute

$$
\begin{aligned}
\frac{1}{2}\left(\frac{\partial}{\partial t}+\Delta\right) \widetilde{R}^{2} & =\widetilde{R}\left(\frac{\partial}{\partial t}+\Delta\right) \widetilde{R}-|d \widetilde{R}|^{2} \\
& \leq 2 \widetilde{R}|h|^{2}+\frac{2 r_{0}}{n} \widetilde{R}^{2} \\
& =2\left(\frac{n-2}{4}|Z|^{2}+\frac{\widetilde{R}^{2}}{n}\right) \widetilde{R}+\frac{2 r_{0} \widetilde{R}^{2}}{n} \\
& =\frac{n-2}{2} \widetilde{R}|Z|^{2}+\frac{2 \widetilde{R}^{3}}{n}+\frac{2 r_{0} \widetilde{R}^{2}}{n} .
\end{aligned}
$$

From the well-known formula for the first variation of the volume form

$$
D(d V)_{g}(\sigma)=\frac{1}{2}(\operatorname{tr} \sigma) d V_{g}
$$

we find that

$$
d V^{\prime}=-(\operatorname{tr} h) d V
$$

Combing this with estimate (3.6) and the Divergence Theorem we find

$$
\begin{aligned}
\frac{1}{2} \frac{d}{d t} \int \widetilde{R}^{2} d V & \leq \int\left\{\frac{n-2}{2} \widetilde{R}|Z|^{2}+\frac{2 \widetilde{R}^{3}}{n}+\frac{2 r_{0} \widetilde{R}^{2}}{n}\right\} d V-\frac{1}{2} \int \widetilde{R}^{2} \operatorname{tr} h d V \\
& =\frac{4-n}{2 n} \int \widetilde{R}^{3} d V+\frac{n-2}{2} \int \widetilde{R}|Z|^{2} d V+\frac{2 r_{0}}{n} \int \widetilde{R}^{2} d V
\end{aligned}
$$

Since $|Z|^{2}=\langle\operatorname{Pr}(\operatorname{Rm}), \operatorname{Pr}(\mathrm{Rm})\rangle$, by the product rule we have that

$$
\frac{\partial}{\partial t}|Z|^{2}=2\left\langle\operatorname{Pr}^{\prime}(\mathrm{Rm}), Z\right\rangle+2\left\langle\operatorname{Pr}\left(\operatorname{Rm}^{\prime}\right), Z\right\rangle+G^{\prime}(Z, Z)
$$

where $G$ denotes the metric on the bundle of 4 -tensors induced by the Riemannian metric. So we will need to estimate $\mathrm{Pr}^{\prime}$.

We begin by fixing a 4 -tensor $S \in \Gamma\left(\Sigma^{2} \Lambda^{2} T^{*} M\right)$. Set $\sigma_{i j}=S_{i k j}{ }^{k}$, and $t=\sigma-$ $\frac{\operatorname{tr} \sigma}{n} g$. By the usual product rule argument we know that $\left(g^{i j}\right)^{\prime}=-g^{i p} g^{j q} g_{p q}^{\prime}=2 h^{i j}$. So

$$
\sigma_{i j}^{\prime}=2 h^{k l} S_{i k j l}
$$

and

$$
(\operatorname{tr} \sigma)^{\prime}=2 h^{i j} \sigma_{i j}+2 g^{i j} h^{k l} S_{i k j l}=4\langle h, \sigma\rangle
$$

Hence

$$
t_{i j}^{\prime}=2 h^{k l} S_{i k j l}+\frac{2 \operatorname{tr} \sigma}{n} h_{i j}-\frac{4\langle\sigma, h\rangle}{n} g_{i j}
$$

By definition $\operatorname{Pr}(S)$ is given by

$$
\operatorname{Pr}(S)=\frac{1}{n-2} t \otimes g
$$

so by the product rule we have

$$
\operatorname{Pr}^{\prime}(S)=\frac{1}{n-2} t^{\prime} \bowtie g-\frac{2}{n-2} t \otimes h .
$$


We wish to estimate

$$
\left\langle\operatorname{Pr}^{\prime}(S), \operatorname{Pr}(S)\right\rangle=\frac{-2}{(n-2)^{2}}\langle t \bowtie h, t \bowtie g\rangle+\frac{1}{(n-2)^{2}}\left\langle t^{\prime} \bowtie g, t \bowtie g\right\rangle .
$$

By the definition of the Nomizu product we have

$$
\langle t \bowtie h, t \bowtie g\rangle=\left(t_{i k} h_{j l}+t_{j l} h_{i k}-t_{i l} h_{j k}-t_{j k} h_{i l}\right)\left(t^{i k} g^{j l}+t^{j l} g^{i k}-t^{i l} g^{j k}-t^{j k} g^{i l}\right)
$$

which when expanded out is a sum of 16 terms, each linear in $h$ and quadratic in $t$. Thus

$$
|\langle t \oslash h, t \oslash g\rangle| \leq c(n)|h||t|^{2} .
$$

Combining a routine index computation with (3.8) shows that

$$
\begin{aligned}
\left\langle t^{\prime} \bowtie g, t \otimes g\right\rangle & =(4 n-8)\left\langle t^{\prime}, t\right\rangle \\
& =4(n-2)\left(2 t^{i j} h^{k l} S_{i k j l}+\frac{2 \operatorname{tr} \sigma}{n} t_{i j} h^{i j}\right)
\end{aligned}
$$

since $t$ is traceless. Substituting (3.10) and (3.11) into (3.9) yields

$$
\left\langle\operatorname{Pr}^{\prime}(S), \operatorname{Pr}(S)\right\rangle \leq c(n)|h||t|^{2}+\frac{8}{n-2} t^{i j} h^{k l} S_{i k j l}+\frac{8 \operatorname{tr} \sigma}{n(n-2)} t_{i j} h^{i j} .
$$

In particular, taking $S=\mathrm{Rm}$ we find that

$$
\left\langle\operatorname{Pr}^{\prime}(\mathrm{Rm}), Z\right\rangle \leq c(n)|h||\stackrel{\circ}{\mathrm{Rc}}|^{2}+\frac{8}{n-2} \mathrm{R}^{i j} h^{k l} R_{i k j l}+\frac{8 s}{n(n-2)}\langle\stackrel{\circ}{\mathrm{Rc}}, h\rangle .
$$

Using (3.4) and the fact that $\left\langle\mathrm{RC}^{\circ}, h\right\rangle=\mid \mathrm{Rc}^{2}$, (3.12) becomes

$$
\left\langle\operatorname{Pr}^{\prime}(\mathrm{Rm}), Z\right\rangle \leq c(n)|h||Z|^{2}+\frac{2 s}{n}|Z|^{2}+\frac{8}{n-2} \mathrm{Rc}^{i j} h^{k l} R_{i k j l} .
$$

In order to estimate the curvature term in 3.13 we use the decomposition of the Riemann tensor into irreducible components. By (2.2) Rm can be written as

$$
\mathrm{Rm}=W+\frac{s}{2 n(n-1)} g \bowtie g+\frac{1}{n-2} \operatorname{Rc} \bowtie g .
$$

By (3.4) we have $\mathrm{Rc}^{i j} h^{k l} Z_{i j k l} \leq c(n)|h||Z|^{2}$. A routine computation combined with (3.4) shows that

$$
\begin{aligned}
\frac{8}{n-2} \mathrm{Rc}^{i j} h^{k l} K_{i k j l} & =-\frac{8 s}{n(n-1)(n-2)}|\stackrel{\circ}{\mathrm{Re}}|^{2} \\
& =-\frac{2 s}{n(n-1)}|Z|^{2} .
\end{aligned}
$$

Finally, $\stackrel{\circ}{\mathrm{Rc}}^{i j} h^{k l} W_{i k j l}=\stackrel{\circ}{\mathrm{Rc}^{i j}} \stackrel{\circ}{\mathrm{Rc}^{k l}} W_{i k j l}$ since $\operatorname{Ric}(W)=0$. Combining these with (3.13) and the observation that $s=\widetilde{R}+r_{0}$ yields

$$
\begin{aligned}
\left\langle\operatorname{Pr}^{\prime}(\mathrm{Rm}), Z\right\rangle \leq & c(n)|h||Z|^{2}+\left(\frac{2}{n}-\frac{2}{n(n-1)}\right)\left(r_{0}+\widetilde{R}\right)|Z|^{2} \\
& +\frac{8}{n-2}\langle\stackrel{\circ}{\operatorname{Rc}} \otimes \stackrel{\circ}{\mathrm{R}}, \widehat{W}\rangle .
\end{aligned}
$$

Finally, we have reached the heart of the argument: the estimate on $\int\left\langle Z^{\prime}, Z\right\rangle$. Here we use that our $\mathrm{Pr}$ is an orthogonal projection to obtain

$$
\left\langle Z^{\prime}, Z\right\rangle=\left\langle\operatorname{Pr}^{\prime}(\mathrm{Rm}), Z\right\rangle+\left\langle\operatorname{Pr}\left(\mathrm{Rm}^{\prime}\right), Z\right\rangle=\left\langle\operatorname{Pr}^{\prime}(\mathrm{Rm}), Z\right\rangle+\left\langle\mathrm{Rm}^{\prime}, Z\right\rangle .
$$


The first term in this sum is estimated in (3.14). Since Rm is obtained from the curvature operator by lowering the third index, we have $\operatorname{Rm}_{i j k l}^{\prime}=\left(g_{k p} R_{i j}{ }^{p}\right)^{\prime}=$ $g_{k p} R_{i j}^{\prime}{ }_{l}-2 h_{k p} R_{i j}{ }^{p}$. So

$$
\int\left\langle\mathrm{Rm}^{\prime}, Z\right\rangle=\int\left\langle R^{\prime}, \widetilde{Z}\right\rangle-2 \int\left\langle h_{k p} R_{i j}{ }^{p}, Z\right\rangle .
$$

From equations (2.15) and (2.16) of [MO] we know that $\int\left\langle R^{\prime}, \widetilde{Z}\right\rangle \leq 0$, so we need only estimate the second term. Again this is done by using the decomposition (2.2). We have

$$
\left\langle h_{k p} R_{i j}{ }^{p} l, Z\right\rangle=h_{k p} Z_{i j}{ }_{l}^{p} Z^{i j k l}+h_{k p} K_{i j}{ }^{p} Z^{i j k l}+h_{k p} W_{i j}{ }^{p} Z^{i j k l} .
$$

The first term is clearly bounded by $c(n)|h||Z|^{2}$. Since $\langle g \bigotimes g, Z\rangle=0$, a routine computation using (3.4), the definitions of $K$ and $Z$, and the fact that $s=\widetilde{R}+r_{0}$ shows that

$$
h_{k p} K_{i j}{ }_{l}{ }_{l} Z^{i j k l}=\frac{n-2}{2 n(n-1)}\left(r_{0}+\widetilde{R}\right)|Z|^{2} .
$$

Finally, using the orthogonality of (2.2), the definition of $Z$, and the fact that $\operatorname{Ric}(W)=0$, we find that

$$
h_{k p} W_{i j}^{p}{ }_{l} Z^{i j k l}=\frac{2}{n-2}\langle\stackrel{\circ}{\operatorname{Rc}} \otimes \stackrel{\circ}{\operatorname{Rc}}, \widehat{W}\rangle .
$$

Thus, combining (3.16) and (3.17) with the estimate 3.14 gives

$$
\begin{aligned}
\int\left\langle Z^{\prime}, Z\right\rangle \leq & c(n) \int|h||Z|^{2}+\left(\frac{2}{n}-\frac{1}{n-1}\right) \int\left(r_{0}+\widetilde{R}\right)|Z|^{2} \\
& +\frac{4}{n-2} \int\langle\stackrel{\circ}{\operatorname{Rc}} \otimes \stackrel{\circ}{\operatorname{Rc}}, \widehat{W}\rangle .
\end{aligned}
$$

By the product rule we have

$$
\left(|Z|^{2}\right)^{\prime}=2\left\langle Z^{\prime}, Z\right\rangle+G^{\prime}(Z, Z),
$$

where $G$ denotes the metric on the bundle of four-tensors induced by the Riemannian metric. Expanding $G^{\prime}(Z, Z)$ in coordinates shows that $G^{\prime}(Z, Z)$ is the sum of four terms, each linear in $h$ and quadratic in $Z$. Hence $G^{\prime}(Z, Z) \leq c(n)|h \| Z|^{2}$. Notice that by (3.5) we have

$$
|Z|^{2} \leq \frac{4}{n-2}|h|^{2}-\frac{4}{n(n-2)} \widetilde{R}^{2} \leq c|h|^{2}
$$

since $|\widetilde{R}|=|\operatorname{tr} h| \leq c(n)|h|$. Combining this with the estimate for $G^{\prime}(Z, Z)$ above and the estimate $(\underline{3.18})$ yields

$$
\begin{aligned}
\frac{1}{2} \frac{d}{d t} \int|Z|^{2} \leq & c(n) \int|h|^{3}+\left(\frac{2}{n}-\frac{1}{n-1}\right) r_{0} \int|Z|^{2} \\
& +\frac{4}{n-2} \int\langle\stackrel{\circ}{\operatorname{Rc}} \otimes \stackrel{\circ}{\operatorname{Rc}}, \widehat{W}\rangle .
\end{aligned}
$$


Finally, combining equation (3.5) with the estimates (3.6) and (3.19) yields

$$
\begin{aligned}
\frac{1}{2} \frac{d}{d t} \int|h|^{2} \leq & c(n) \int|h|^{3}+\left(\frac{2}{n}-\frac{1}{n-1}\right) r_{0} \int\left(|h|^{2}-\frac{\widetilde{R}^{2}}{n}\right) \\
& +\frac{4-n}{2 n^{2}} \int \widetilde{R}^{3}+\frac{n-2}{2 n} \int \widetilde{R}|Z|^{2}+\frac{2 r_{0}}{n^{2}} \int \widetilde{R}^{2}+\int\langle\stackrel{\circ}{\operatorname{R} c} \otimes \stackrel{\circ}{\operatorname{R} c}, \widehat{W}\rangle \\
\leq & c(n) \int|h|^{3}+\left(\frac{2}{n}-\frac{1}{n-1}\right) r_{0} \int|h|^{2} \\
& +\frac{r_{0}}{n(n-1)} \int \widetilde{R}^{2}+\int\langle\stackrel{\circ}{\operatorname{Rc}} \otimes \stackrel{\circ}{\operatorname{Re}, \widehat{W}\rangle} \\
\leq & c(n) \int|h|^{3}+\left(\frac{2}{n}-\frac{1}{n-1}\right) r_{0} \int|h|^{2}+\int\langle\operatorname{Rc} \otimes \stackrel{\circ}{\operatorname{Rc}, \widehat{W}\rangle},
\end{aligned}
$$

where in the last two lines we have used the fact that $|Z|^{2} \leq c(n)|h|^{2}$ and the hypothesis that $r_{0}<0$.

Proof of Claim A for $n=3$. As in Min-Oo's work, the inequality (3.1) is the key to proving the long-term existence and convergence of the Ricci flow (1.2). Recall that in the three-dimensional case the Weyl tensor is identically zero. So (3.1) becomes

$$
\frac{1}{2} \frac{d}{d t} \int|h|^{2} \leq \frac{1}{6} r_{0} \int|h|^{2}+c \int|h|^{3} .
$$

The Moser iteration argument given in section three of [MO] can be applied, using our corrected inequality, with only a minor cosmetic change: the inequality (A1) of [M] must be slightly modified. This change is necessitated by the fact that we have a factor of $\frac{1}{6}$ in our inequality where Min-Oo has a factor of $\frac{2}{3}$ (cf. (2.21) of [MO]). We must modify the form of the assumption (A1) because Min-Oo's argument requires the same exponential factor in assumption (A1) as in the estimate of the $L^{2}$-norm of $h$ (equation (3.11) of [MO]) derived from it.

As in Section 3 of $\mathrm{MO}$, let

$$
H(t)=\max _{|X|_{t}=1}|h(X, X)|=\max _{|X|_{t}=1}|\operatorname{Rc}(X, X)+2 \lambda|
$$

where $\lambda=-r_{0} / 2 n>0$ and $|\cdot|_{t}$ denotes the norm associated with the metric $g_{t}$ at time $t$. We claim that the inequality

$$
H(t)^{2} \leq 16 n^{2} \lambda^{2} \epsilon \exp \left(-\frac{3}{2} \lambda t\right)
$$

will suffice as a replacement for Min-Oo's (A1). To see that this is the case, first note that the same maximum principle argument given by Min-Oo shows that there is some maximal time interval $[0,2 T]$ on which $\left(\mathrm{A1}^{\prime}\right)$ holds. Noting that

$$
\max |h(t)|^{2} \leq n H(t)^{2}
$$

we see from 3.20 that

$$
\begin{aligned}
\left(\int|h|^{2}\right)^{\prime} & \leq-2 \lambda \int|h|^{2}+c H(t) \int|h|^{2} \\
& \leq-2 \lambda \int|h|^{2}+c \lambda \sqrt{\epsilon} \int|h|^{2} \leq-\frac{3}{2} \lambda \int|h|^{2}
\end{aligned}
$$


on $[0,2 T]$ provided that $\epsilon$ is chosen such that $c \sqrt{\epsilon}<\frac{1}{2}$. Integrating both sides of (3.21) gives

$$
\|h\|_{L^{2}}^{2} \leq\|h(0)\|_{L^{2}}^{2} \exp \left(-\frac{3}{2} \lambda t\right)
$$

for all $t \in[0,2 T]$. Notice that the exponential factor in this last inequality is the same as that in our $\left(\mathrm{A}^{\prime}\right)$. The Moser iteration argument given in Section 3 of [MO] now carries through with our (A1') replacing Min-Oo's (A1).

\section{Conclusion}

Our adaptation of Min-Oo's proof makes quite extensive use of the fact that the Weyl tensor vanishes on 3-manifolds: we need that the Weyl tensor is identically zero all along the flow. A reasonable first step in trying to generalize our proof to higher dimensions would be trying to show that, on a manifold of arbitrary dimension, the condition of having zero Weyl tensor is preserved along the flow (1.2). If this held, it would yield a proof of a modified version of Claim A, in arbitrary dimension, where $\mathcal{M}^{-}(n, \Lambda)$ replaced by the smaller class of all metrics in $\mathcal{M}^{-}(n, \Lambda)$ which are also locally conformally flat.

Unfortunately, we have not been able to find a proof that the zero Weyl tensor condition is preserved along the flow. A laborious computation shows that the square-norm of the Weyl tensor evolves according to

$$
\left(\frac{\partial}{\partial t}+\Delta\right)|W|^{2} \leq f_{1}|W|^{2}+c(n)|W|^{3}+f_{2}\langle\stackrel{\circ}{\operatorname{Rc}} \otimes \stackrel{\circ}{\operatorname{Rc}}, \widehat{W}\rangle,
$$

where $f_{1}$ and $f_{2}$ are continuous functions on $M \times[0, T)$ determined from $r_{0}$, the norm of the traceless Ricci tensor, and the scalar curvature. (Here $[0, T$ ) is the interval of existence for the flow.) It is interesting that what prevents us from applying the usual maximum principle to conclude that $|W|^{2}$ is zero along the flow if it vanishes initially is the presence of the $\langle\stackrel{\circ}{\mathrm{R} c} \otimes \stackrel{\circ}{\mathrm{R}}, \widehat{W}\rangle$ term, the same troublesome term which arises in (3.1) in dimensions greater than three. This suggests that this term may have some significance worthy of further study.

\section{REFERENCES}

[Be] A. Besse, Einstein Manifolds, Springer-Verlag, New York, 1987. MR 88f:53087

[Ha] R. Hamilton, Three-manifolds with positive Ricci curvature, J. D. Geo. 17 (1982) 255-306. MR 84a:53050

[MO] M. Min-Oo, Almost Einstein manifolds of negative Ricci curvature, J. Diff. Geo. 32 (1990) 457-472. MR 91g:53047

[Ye] R. Ye, Ricci flow, Einstein metrics and space forms, Trans. Amer. Math. Soc. 338 (1993) 871-896. MR 93j:58029

4746 19th Ave NE, \#5, Seattle, Washington 98105

E-mail address: skinner@math.washington.edu 